\title{
Finite Difference and Sinc-Collocation Approximations to a Class of Fractional Diffusion-Wave Equations
}

\author{
Zhi Mao, ${ }^{1,2}$ Aiguo Xiao, ${ }^{1}$ Zuguo Yu, ${ }^{1}$ and Long Shi ${ }^{1}$ \\ ${ }^{1}$ Hunan Key Laboratory for Computation and Simulation in Science and Engineering, Xiangtan University, \\ Xiangtan, Hunan 411105, China \\ ${ }^{2}$ Mathematics and Information Engineering Department, Tongren University, Tongren, Guizhou 554300, China \\ Correspondence should be addressed to Zhi Mao; mzxtu1982@163.com
}

Received 10 February 2014; Revised 30 May 2014; Accepted 9 June 2014; Published 2 July 2014

Academic Editor: Nazim I. Mahmudov

Copyright (c) 2014 Zhi Mao et al. This is an open access article distributed under the Creative Commons Attribution License, which permits unrestricted use, distribution, and reproduction in any medium, provided the original work is properly cited.

\begin{abstract}
We propose an efficient numerical method for a class of fractional diffusion-wave equations with the Caputo fractional derivative of order $\alpha$. This approach is based on the finite difference in time and the global sinc collocation in space. By utilizing the collocation technique and some properties of the sinc functions, the problem is reduced to the solution of a system of linear algebraic equations at each time step. Stability and convergence of the proposed method are rigorously analyzed. The numerical solution is of $3-\alpha$ order accuracy in time and exponential rate of convergence in space. Numerical experiments demonstrate the validity of the obtained method and support the obtained theoretical results.
\end{abstract}

\section{Introduction}

Fractional diffusion-wave equations have attracted considerable attention as generalization of the classical diffusion/wave equation by replacing the integer-order time derivative with a fractional derivative of order $\alpha(1<\alpha<2)$ [1]. It can be derived from the physical case of a general random timedependent velocity function equipped with an algebraic timecorrelation function (but with a Gaussian space correlation) [2]. Owing to the anomalous superdiffusion of particles, many electromagnetic, acoustic, mechanical, and biological responses can be modelled accurately by fractional diffusionwave equations, for example, the propagation of stress waves in viscoelastic solids [3, 4]. There exist some analytical methods to find exact solutions of fractional diffusion-wave equations [5-9]. However, it is usually difficult or even impossible to achieve the exact solutions in many cases, and ones have to resort to numerical methods.

Compared with the considerable literature on numerical solutions of fractional diffusion equations [10-15], only a few works have been carried out in the numerical methods of fractional diffusion-wave equations. The homotopy analysis method [16] and the Adomian decomposition method [17] were used to construct analytical approximate solutions of fractional diffusion-wave equations, respectively. Sun and $\mathrm{Wu}$ [18] presented a full discrete difference scheme for the initial-boundary-value problem of a diffusion-wave equation by introducing two new variables and obtained the stability and convergence properties by using energy method. In [19], under the weak smoothness conditions, two finite difference schemes with first-order accuracy in temporal direction and second-order accuracy in spatial direction were proposed to solve the same problem. Murillo and Yuste [20] developed an explicit difference method where the L2 discretization formula is used for solving fractional diffusionwave equations. Due to the nonlocal nature of fractional derivatives, all previous solutions have to be saved to compute the solution at the current time level. It makes the storage very expensive when low-order methods are employed for spatial discretization.

In this paper, we propose a numerical method mixing finite difference with sinc collocation to solve a class of fractional diffusion-wave equations. The sinc method is widely used to solve integral, integro-differential, ordinary differential, and partial differential equations [21-27]. As far as we know, there are very limited papers on solving fractional 
differential equation by the sinc method $[28,29]$. The present method has great advantage in storage requirement because the sinc-collocation method needs fewer grid points to produce highly accurate solution when it is compared with a low-order method.

The remainder of this paper is organized as follows. In Section 2, we introduce some necessary definitions and relevant results for developing this method. Section 3 is devoted to the finite difference and sinc-collocation discretization for the fractional diffusion-wave equations. As a result, a system of linear algebraic equations is formed, and the solutions of the considered problems are obtained. Section 4 is concerned with the stability and convergence analysis. In Section 5, some numerical experiments are given to demonstrate the effectiveness of the proposed method and the obtained theoretical results. A brief conclusion ends this paper in the final section.

\section{Notations and Some Preliminary Results}

In this section, we introduce some basic definitions and relevant results of the fractional calculus $[30,31]$ and sinc functions.

Definition 1 (see [32]). Let $\alpha \in \mathscr{R}_{+}$. The operator $J_{a}^{\alpha}$ defined on $L_{1}[a, b]$ by

$$
J_{a}^{\alpha} f(t)=\frac{1}{\Gamma(\alpha)} \int_{a}^{t}(t-s)^{\alpha-1} f(s) d s
$$

for $a \leq t \leq b$ is called the Riemann-Liouville fractional integral operator of order $\alpha$. For $\alpha=0$, one sets $J_{a}^{0}:=I$, that is, the identity operator.

Definition 2 (see [32]). Let $\alpha \in \mathscr{R}_{+}$and $n=\lceil\alpha\rceil$. The Caputo fractional differential operator ${ }^{C} D_{a}^{\alpha}$ for $a \leq t \leq b$ is defined as

$$
\begin{aligned}
{ }^{C} D_{a}^{\alpha} f(t) & =J_{a}^{n-\alpha} D^{n} f(t) \\
& =\frac{1}{\Gamma(n-\alpha)} \int_{a}^{t}(t-s)^{n-\alpha-1} f^{(n)}(s) d s .
\end{aligned}
$$

The sinc functions and its properties are discussed thoroughly in $[21,23]$. For any $h>0$, the translated sinc functions with equidistant space nodes are given as

$$
S(k, h)(z)=\operatorname{sinc}\left(\frac{z-k h}{h}\right), \quad k=0, \pm 1, \pm 2, \ldots,
$$

where the sinc functions are defined on the whole real line by

$$
\operatorname{sinc}(x)= \begin{cases}\frac{\sin (\pi x)}{\pi x}, & x \neq 0 \\ 1, & x=0\end{cases}
$$

If $f$ is defined on $\mathbb{R}$, then for any $h>0$ the series

$$
C(f, h)(z)=\sum_{k=-\infty}^{\infty} f(k h) S(k, h)(z)
$$

is called the Whittaker cardinal expansion of $f$ whenever this series converges. $f$ can be approximated by truncating (5). To construct our needed approximations on the interval $[a, b]$, we choose

$$
\phi(x)=\ln \left(\frac{x-a}{b-x}\right)
$$

which maps a finite interval $[a, b]$ onto $\mathbb{R}$. $\phi$ is a conformal map which maps the eye-shaped domain in the complex $z$ plane

$$
D_{E}=\left\{z \in \mathbb{C}:\left|\arg \left(\frac{z-a}{b-z}\right)\right|<d \leq \frac{\pi}{2}\right\}
$$

onto the infinite strip $D_{S}$ of the complex $\widetilde{z}$-plane

$$
D_{S}=\left\{\widetilde{z} \in \mathbb{C}:|\operatorname{Im}(\widetilde{z})|<d \leq \frac{\pi}{2}\right\} .
$$

The basis functions on $[a, b]$ are taken to be the composite translated sinc functions

$$
S_{\phi}(k, h)(x)=S(k, h)(\phi(x))=\operatorname{sinc}\left(\frac{\phi(x)-k h}{h}\right) .
$$

Thus one may define the inverse image of the equidistant space node $\{i h\}$ as

$$
x_{i}=\phi^{-1}(i h)=\frac{a+b e^{i h}}{1+e^{i h}}, \quad i=0, \pm 1, \pm 2, \ldots
$$

The class of functions such that the known exponential convergence rate exists for the sinc interpolation is denoted by $B\left(D_{E}\right)$ and defined in the following.

Definition 3 (see $[21,23])$. Let $B\left(D_{E}\right)$ be the class of functions $f$ which are analytic in $D_{E}$ and satisfy

$$
\int_{\phi^{-1}(x+L)}|f(z) d z| \longrightarrow 0, \quad x \longrightarrow \pm \infty,
$$

where $L=\{i v:|v|<d \leq(\pi / 2)\}$ and

$$
\int_{\partial D_{E}}|f(z) d z|<\infty
$$

on the boundary of $D_{E}$ (denoted $\left.\partial D_{E}\right)$.

The following theorem gives the error which results from differentiating the truncated cardinal series.

Theorem 4 (see $[21,22])$. Let $\phi^{\prime} f / g \in B\left(D_{E}\right)$ and let

$$
\sup _{x \in[a, b],-\pi / h \leq t \leq \pi / h}\left|\left(\frac{\mathrm{d}}{\mathrm{d} x}\right)^{n}\left[g(x) e^{i t \phi(x)}\right]\right| \leq C_{1} h^{-n},
$$

for $n=0,1, \ldots, m$; here $g$ is a weight function and $C_{1}$ is a constant depending only on $m, \phi$, and $g$. Assume that there exists a constant $C_{2}$ such that

$$
\frac{f(x)}{g(x)} \leq C_{2} \frac{e^{\beta \phi(x)}}{\left[1+e^{\phi(x)}\right]^{2 \beta}} ;
$$


here $\beta$ is a positive constant. Then taking $h=\sqrt{\pi d / \beta N}$, one has

$$
\begin{aligned}
& \left|\frac{\mathrm{d}^{n} f(x)}{\mathrm{d} x^{n}}-\sum_{k=-N}^{N} \frac{f\left(x_{k}\right)}{g\left(x_{k}\right)} \frac{\mathrm{d}^{n}}{\mathrm{~d} x^{n}}\left[g(x) S_{\phi}(k, h)(x)\right]\right| \\
& \quad \leq C_{3} N^{(m+1) / 2} \exp (-\sqrt{\pi d \beta N})
\end{aligned}
$$

for all $n=0,1, \ldots, m$; here $C_{3}$ is a constant depending only on $m, \phi, g, d, \beta$, and $f$.

The above expressions show that the sinc interpolation on $B\left(D_{E}\right)$ converges exponentially. We also require the following derivatives of the composite translated sinc functions evaluated at the nodes:

$$
\begin{gathered}
\delta_{k, i}^{(0)}=\left.\left[S_{\phi}(k, h)(x)\right]\right|_{x=x_{i}}= \begin{cases}1, & k=i, \\
0, & k \neq i .\end{cases} \\
\delta_{k, i}^{(1)}=\left.\frac{\mathrm{d}}{\mathrm{d} \phi}\left[S_{\phi}(k, h)(x)\right]\right|_{x=x_{i}}= \begin{cases}0, & k=i, \\
\frac{(-1)^{i-k}}{(i-k) h}, & k \neq i .\end{cases} \\
\delta_{k, i}^{(2)}=\left.\frac{\mathrm{d}^{2}}{\mathrm{~d} \phi^{2}}\left[S_{\phi}(k, h)(x)\right]\right|_{x=x_{i}}= \begin{cases}\frac{-\pi^{2}}{3 h^{2}}, & k=i, \\
\frac{-2(-1)^{i-k}}{(i-k)^{2} h^{2}}, & k \neq i .\end{cases}
\end{gathered}
$$

\section{The Finite Difference and Sinc-Collocation Discretization}

We consider the following fractional diffusion-wave equations with a nonhomogeneous field [33]:

$$
\frac{1}{c} \frac{\partial^{\alpha} u(x, t)}{\partial t^{\alpha}}=\frac{\partial^{2} u(x, t)}{\partial x^{2}}+\frac{1}{K} f(x, t), \quad a<x<b, t>0
$$

with the initial conditions

$$
u(x, 0)=\varphi(x), \quad \frac{\partial u(x, 0)}{\partial t}=\psi(x), \quad a<x<b
$$

and the boundary conditions

$$
u(a, t)=0, \quad u(b, t)=0, \quad t>0,
$$

where $x \in[a, b]$ and $t>0$ are space and time variables, $c$ and $K$ are constants, and $f(x, t)$ denotes the field variable. Here the time-fractional derivative is defined as the Caputo fractional derivative, and $1<\alpha<2$. Many authors refer to the fractional equation (19) as the fractional diffusion-wave equation when $1<\alpha<2$, which is expected to interpolate the diffusion equation and the wave equation $[7,18]$.

3.1. Temporal Discretization by a Finite Difference Scheme. First, we derive a finite difference scheme for temporal discretization of this equation. Let $t_{k}:=k \tau, k=0,1,2, \ldots$, where $\tau$ is the time stepsize. In order to discretize the time-fractional derivative by using a finite difference approximation $[18,34]$, we introduce the following lemmas.

Lemma 5 (see [18]). Suppose $f(t) \in C^{2}\left[0, t_{n}\right]$. Then

$$
\begin{aligned}
& \mid \int_{0}^{t_{n}} \frac{f^{\prime}(t) d t}{\left(t_{n}-t\right)^{\alpha-1}}-\frac{\tau^{1-\alpha}}{2-\alpha} \\
& \quad \times\left[b_{0} f\left(t_{n}\right)-\sum_{k=1}^{n-1}\left(b_{k-1}-b_{k}\right) f\left(t_{n-k}\right)-b_{n-1} f\left(t_{0}\right)\right] \mid \\
& \quad \leq \frac{1}{2-\alpha}\left[\frac{2-\alpha}{12}+\frac{2^{3-\alpha}}{3-\alpha}-\left(1+2^{1-\alpha}\right)\right] \max _{0 \leq t \leq t_{n}}\left|f^{\prime \prime}(t)\right| \tau^{3-\alpha},
\end{aligned}
$$

where $1<\alpha<2$ and

$$
b_{k}=(k+1)^{2-\alpha}-k^{2-\alpha}, \quad k=0,1,2, \ldots
$$

It is direct to check that

$$
1=b_{0}>b_{1}>\cdots>b_{n}>0, \quad b_{n} \longrightarrow 0 \text { as } n \longrightarrow \infty .
$$

Let

$$
\phi(x, t)=\frac{\partial^{\alpha} u(x, t)}{\partial t^{\alpha}}=\frac{1}{\Gamma(2-\alpha)} \int_{0}^{t} \frac{\partial^{2} u(x, s)}{\partial s^{2}} \frac{d s}{(t-s)^{\alpha-1}},
$$

and define the temporal grid functions

$$
U^{k}=u\left(x, t_{k}\right), \quad \Phi^{k}=\phi\left(x, t_{k}\right), \quad k=0,1,2, \ldots
$$

By the Taylor expansion, it follows that

$$
\begin{gathered}
U_{t}^{k-(1 / 2)}=\frac{1}{\tau}\left(U^{k}-U^{k-1}\right)+r_{1}^{k} \tau^{2}, \\
\frac{1}{c} \Phi^{k-(1 / 2)}=\left(U^{k-(1 / 2)}\right)_{x x}+\frac{1}{K} f^{k-(1 / 2)}+r_{2}^{k} \tau^{2},
\end{gathered}
$$

where $r_{1}^{k}, r_{2}^{k}$ are constants. Based on Lemma 5, one has

$$
\begin{aligned}
\Phi^{k}= & \frac{\tau^{1-\alpha}}{\Gamma(3-\alpha)}\left[b_{0} U_{t}^{k}-\sum_{l=1}^{k-1}\left(b_{l-1}-b_{l}\right) U_{t}^{k-l}-b_{k-1} U_{t}^{0}\right] \\
& +O\left(\tau^{3-\alpha}\right), \quad k=1,2,3, \ldots
\end{aligned}
$$

Hence,

$$
\begin{aligned}
\Phi^{k-(1 / 2)}= & \frac{1}{2}\left(\Phi^{k}+\Phi^{k-1}\right) \\
= & \frac{\tau^{1-\alpha}}{\Gamma(3-\alpha)} \\
& \times\left[b_{0} U_{t}^{k-(1 / 2)}-\sum_{l=1}^{k-1}\left(b_{l-1}-b_{l}\right) U_{t}^{k-l-(1 / 2)}-b_{k-1} U_{t}^{0}\right] \\
& +r_{3}^{k} \tau^{3-\alpha},
\end{aligned}
$$


where $r_{3}^{k}$ are constants. Let $\delta_{t} U^{k-(1 / 2)}=(1 / \tau)\left(U^{k}-U^{k-1}\right)$. In view of (27), one has

$$
\begin{aligned}
\Phi^{k-(1 / 2)} & \\
= & \frac{\tau^{1-\alpha}}{\Gamma(3-\alpha)} \\
& \times\left[b_{0} \delta_{t} U^{k-(1 / 2)}-\sum_{l=1}^{k-1}\left(b_{l-1}-b_{l}\right) \delta_{t} U^{k-l-(1 / 2)}-b_{k-1} U_{t}^{0}\right] \\
& +\frac{\tau^{3-\alpha}}{\Gamma(3-\alpha)}\left[b_{0} r_{1}^{k}-\sum_{l=1}^{k-1}\left(b_{l-1}-b_{l}\right) r_{1}^{k-l}\right]+r_{3}^{k} \tau^{3-\alpha} .
\end{aligned}
$$

Then substituting (31) into (28) and observing $U_{t}^{0}=\psi(x)$, one obtains

$$
\begin{aligned}
& \frac{\tau^{1-\alpha}}{c \Gamma(3-\alpha)}\left[b_{0} \delta_{t} U^{k-(1 / 2)}-\sum_{l=1}^{k-1}\left(b_{l-1}-b_{l}\right) \delta_{t} U^{k-l-(1 / 2)}-b_{k-1} \psi\right] \\
& \quad=\left(U^{k-(1 / 2)}\right)_{x x}+\frac{1}{K} f^{k-(1 / 2)}+R_{T}^{k},
\end{aligned}
$$

where

$$
\begin{aligned}
R_{T}^{k}= & -\frac{\tau^{3-\alpha}}{c \Gamma(3-\alpha)}\left[b_{0} r_{1}^{k}-\sum_{l=1}^{k-1}\left(b_{l-1}-b_{l}\right) r_{1}^{k-l}\right]-\frac{1}{c} r_{3}^{k} \tau^{3-\alpha} \\
& +r_{2}^{k} \tau^{2} .
\end{aligned}
$$

It follows from (32) that one can construct easily the following semidiscrete finite difference scheme for (19):

$$
\begin{aligned}
& \frac{\tau^{1-\alpha}}{c \Gamma(3-\alpha)}\left[b_{0} \delta_{t} u^{k-(1 / 2)}-\sum_{l=1}^{k-1}\left(b_{l-1}-b_{l}\right) \delta_{t} u^{k-l-(1 / 2)}-b_{k-1} \psi\right] \\
& \quad=\left(u^{k-(1 / 2)}\right)_{x x}+\frac{1}{K} f^{k-(1 / 2)},
\end{aligned}
$$

which is equivalent to

$$
\begin{aligned}
\frac{1}{c \tau^{\alpha} \Gamma(3-\alpha)}\left(\Delta_{1}+\Delta_{2}\right)= & \frac{1}{2}\left[\left(u^{k}\right)_{x x}+\left(u^{k-1}\right)_{x x}\right] \\
& +\frac{1}{K} f^{k-(1 / 2)}, \quad k=2,3, \ldots,
\end{aligned}
$$

where

$$
\begin{aligned}
& \Delta_{1}=b_{0} u^{k}-\left(2 b_{0}-b_{1}\right) u^{k-1}, \\
& \Delta_{2}=\sum_{l=2}^{k-1}\left(b_{l-2}-2 b_{l-1}+b_{l}\right) u^{k-l}+\left(b_{k-2}-b_{k-1}\right) u^{0}-b_{k-1} \tau \psi .
\end{aligned}
$$

According to (33), this scheme is of $(3-\alpha)$-order accuracy. A rigorous analysis of the convergence rate will be provided later. The above scheme can be rewritten into

$$
\begin{aligned}
& \frac{b_{0}}{c \tau^{\alpha} \Gamma(3-\alpha)} u^{k}-\frac{1}{2}\left(u^{k}\right)_{x x} \\
& =\frac{2 b_{0}-b_{1}}{c \tau^{\alpha} \Gamma(3-\alpha)} u^{k-1}-\frac{1}{c \tau^{\alpha} \Gamma(3-\alpha)} \Delta_{2}+\frac{1}{2}\left(u^{k-1}\right)_{x x} \\
& \quad+\frac{1}{K} f^{k-(1 / 2)} .
\end{aligned}
$$

Specially, for the case $k=1$, the scheme simply reads

$$
\begin{aligned}
& \frac{b_{0}}{c \tau^{\alpha} \Gamma(3-\alpha)} u^{1}-\frac{1}{2}\left(u^{1}\right)_{x x} \\
& =\frac{b_{0}}{c \tau^{\alpha} \Gamma(3-\alpha)}\left(u^{0}+\tau \psi\right)+\frac{1}{2}\left(u^{0}\right)_{x x}+\frac{1}{K} f^{(1 / 2)} .
\end{aligned}
$$

So (37) and (38) together with the initial condition

$$
u^{0}=u(x, 0)=\varphi(x), \quad a<x<b
$$

and the boundary conditions

$$
u_{a}^{k}=u\left(a, t_{k}\right)=0, \quad u_{b}^{k}=u\left(b, t_{k}\right)=0, \quad k \geq 1
$$

form a complete semidiscrete problem.

3.2. Space Discretization by the Sinc-Collocation Method. Next we consider space discretization to (37) by the sinccollocation method. We select the collocation points $x_{i}$ by (10). The space discretization proceeds by approximating the solution based on the composite translated sinc functions (9)

$$
u^{k} \simeq u_{N}^{k}=\sum_{j=-n}^{n} u_{j}^{k} S_{\phi}(j, h)(x) .
$$

The unknown parameters $u_{j}^{k}$ will be determined by the collocation method. It is noted that the approximation in (41) satisfies the boundary conditions in $(40)$ since $S_{\phi}(j, h)(x)$, $j=-n,-n+1, \ldots, n$ are zero when $x$ tends to $a$ and $b$. 
Now substituting (41) into (37) and collocating in $2 n+1$ points $x_{i}$, we obtain

$$
\begin{aligned}
& \frac{b_{0}}{c \tau^{\alpha} \Gamma(3-\alpha)} \sum_{j=-n}^{n} u_{j}^{k} S_{\phi}(j, h)\left(x_{i}\right)-\frac{1}{2} \sum_{j=-n}^{n} u_{j}^{k} S_{\phi}^{\prime \prime}(j, h)\left(x_{i}\right) \\
& =\frac{2 b_{0}-b_{1}}{c \tau^{\alpha} \Gamma(3-\alpha)} \sum_{j=-n}^{n} u_{j}^{k-1} S_{\phi}(j, h)\left(x_{i}\right) \\
& +\frac{1}{2} \sum_{j=-n}^{n} u_{j}^{k-1} S_{\phi}^{\prime \prime}(j, h)\left(x_{i}\right)-\frac{1}{\tau^{\alpha} \Gamma(3-\alpha)} \\
& \times\left[\sum_{l=2}^{k-1}\left(b_{l-2}-2 b_{l-1}+b_{l}\right)\right. \\
& \quad \times \sum_{j=-n}^{n} u_{j}^{k-l} S_{\phi}(j, h)\left(x_{i}\right)+\left(b_{k-2}-b_{k-1}\right) u^{0}\left(x_{i}\right) \\
& \left.\quad-\tau b_{k-1} \psi\left(x_{i}\right)\right]+\frac{1}{K} f^{k-(1 / 2)}\left(x_{i}\right),
\end{aligned}
$$

where $i=-n,-n+1, \ldots, n$. Based on (17) and (18), we let

$$
q_{j i}:=S_{\phi}^{\prime \prime}(j, h)\left(x_{i}\right)=\phi^{\prime \prime}\left(x_{i}\right) \delta_{j, i}^{(1)}+\left(\phi^{\prime}\left(x_{i}\right)\right)^{2} \delta_{j, i}^{(2)} .
$$

Hence, (42) is reduced immediately by (16) and (43) to

$$
\begin{aligned}
& \frac{b_{0}}{c \tau^{\alpha} \Gamma(3-\alpha)} u_{i}^{k}-\frac{1}{2} \sum_{j=-n}^{n} q_{j i} u_{j}^{k} \\
& =\frac{2 b_{0}-b_{1}}{c \tau^{\alpha} \Gamma(3-\alpha)} u_{i}^{k-1}-\frac{1}{c \tau^{\alpha} \Gamma(3-\alpha)} \\
& \times\left[\sum_{l=2}^{k-1}\left(b_{l-2}-2 b_{l-1}+b_{l}\right) u_{i}^{k-l}\right. \\
& \left.+\left(b_{k-2}-b_{k-1}\right) u^{0}\left(x_{i}\right)-\tau b_{k-1} \psi\left(x_{i}\right)\right] \\
& +\frac{1}{2} \sum_{j=-n}^{n} q_{j i} u_{j}^{k-1}+\frac{1}{K} f^{k-(1 / 2)}\left(x_{i}\right),
\end{aligned}
$$

where $i=-n,-n+1, \ldots, n$. To obtain a matrix representation of the above equation, we let

$$
\begin{gathered}
A=\frac{b_{0}}{c \tau^{\alpha} \Gamma(3-\alpha)} I_{(2 n+1) \times(2 n+1)}, \\
B=\frac{1}{2}\left(q_{j i}\right)_{(2 n+1) \times(2 n+1)}, \\
C^{(1)}=\frac{2 b_{0}-b_{1}}{c \tau^{\alpha} \Gamma(3-\alpha)} I_{(2 n+1) \times(2 n+1)}, \\
C^{(l)}=-\frac{b_{l-2}-2 b_{l-1}+b_{l}}{c \tau^{\alpha} \Gamma(3-\alpha)} I_{(2 n+1) \times(2 n+1)}, \\
C^{(k)}=-\frac{b_{k-2}-b_{k-1}}{c \tau^{\alpha} \Gamma(3-\alpha)} I_{(2 n+1) \times(2 n+1)}, \\
D=\frac{b_{k-1}}{c \tau^{\alpha-1} \Gamma(3-\alpha)} I_{(2 n+1) \times(2 n+1)}, \\
\mathbb{U}^{k}=\left[u_{-n}^{k}, u_{-n+1}^{k}, \ldots, u_{n}^{k}\right]^{T}, \\
\Psi=\left[\psi\left(x_{-n}\right), \psi\left(x_{-n+1}\right), \ldots, \psi\left(x_{n}\right)\right]^{T}, \\
F^{k-(1 / 2)} \quad \frac{1}{K}\left[f^{k-(1 / 2)}\left(x_{-n}\right), f^{k-(1 / 2)}\left(x_{-n+1}\right), \ldots, f^{k-(1 / 2)}\left(x_{n}\right)\right]^{T},
\end{gathered}
$$

where the matrix $I$ is the identity matrix and $l=2,3, \ldots, k-1$. Therefore, at each time step, we get the following system of $2 n+1$ linear equations with $2 n+1$ unknown parameters $u_{j}^{k}$, and this system can be expressed in a matrix form

$$
Q \mathbb{U}^{k}=P \text {, }
$$

where

$$
Q=A-B,
$$

$$
P=B \bigcup^{k-1}+\sum_{l=1}^{k} C^{(l)} \bigcup^{k-l}+D \Psi+F^{k-(1 / 2)}
$$

For the initial condition (39), we have

$$
\mathbb{U}^{0}=\left[\varphi\left(x_{-n}\right), \varphi\left(x_{-n+1}\right), \ldots, \varphi\left(x_{n}\right)\right]^{T} .
$$

Consequently (46) can be solved easily for the unknown coefficients $\mathbb{U}^{k}$. Hence the approximation solution given in (41) can be obtained.

\section{Stability and Convergence Analysis of the Derived Method}

To analyse the stability and convergence of the derived method, the inner product is defined by $\langle u, v\rangle=\int_{a}^{b} u v d x$ with the corresponding norm $\|u\|=(u, u)^{(1 / 2)}$, which will be used thereafter. We first give the following lemmas. 
Lemma 6 (see [18]). For any $Q=Q_{1}, Q_{2}, Q_{3}, \ldots$ and $v$, here $Q_{i}, v \in L^{2}(a, b)$, it can be verified that

$$
\begin{array}{r}
\sum_{i=1}^{k}\left\langle b_{0} Q_{i}-\sum_{l=1}^{i-1}\left(b_{l-1}-b_{l}\right) Q_{i-l}-b_{i-1} v, Q_{i}\right\rangle \\
\geq \frac{1}{2}(2-\alpha) k^{1-\alpha} \sum_{i=1}^{k}\left\|Q_{i}\right\|^{2}-\frac{1}{2} k^{2-\alpha}\|v\|^{2} \\
k=1,2,3, \ldots
\end{array}
$$

where $b_{l}$ is defined in (23).

Lemma 7. Suppose that $u_{N}^{k}, k=1,2,3, \ldots$, satisfy

$$
\begin{gathered}
\frac{\tau^{1-\alpha}}{c \Gamma(3-\alpha)}\left[b_{0} \delta_{t} u_{N}^{k-(1 / 2)}-\sum_{l=1}^{k-1}\left(b_{l-1}-b_{l}\right) \delta_{t} u_{N}^{k-l-(1 / 2)}-b_{k-1} v\right] \\
=\left(u_{N}^{k-(1 / 2)}\right)_{x x}+f^{k-(1 / 2)} \\
u_{N}^{0}=\varphi, \quad 0<x<1 \\
u_{N}^{k}(a)=0, \quad u_{N}^{k}(b)=0
\end{gathered}
$$

Then one has the estimate

$$
\begin{aligned}
\left\|\left(u_{N}^{k}\right)_{x}\right\|^{2} \leq & \left\|\left(u_{N}^{0}\right)_{x}\right\|^{2}+\frac{t_{k}^{2-\alpha}}{c \Gamma(3-\alpha)}\|v\|^{2} \\
& +c \Gamma(2-\alpha) t_{k}^{\alpha-1} \tau \sum_{l=1}^{k}\left\|f^{k-(1 / 2)}\right\|^{2} .
\end{aligned}
$$

Proof. Based on (50), we have

$$
\begin{gathered}
\frac{\tau^{1-\alpha}}{c \Gamma(3-\alpha)} \sum_{k=1}^{m}\left\langle b_{0} \delta_{t} u_{N}^{k-(1 / 2)}-\sum_{l=1}^{k-1}\left(b_{l-1}-b_{l}\right) \delta_{t} u_{N}^{k-l-(1 / 2)}\right. \\
\left.-b_{k-1} v, \tau \delta_{t} u_{N}^{k-(1 / 2)}\right\rangle \\
=\sum_{k=1}^{m}\left(\left(u_{N}^{k-(1 / 2)}\right)_{x x}, \tau \delta_{t} u_{N}^{k-(1 / 2)}\right) \\
+\sum_{k=1}^{m}\left(f^{k-(1 / 2)}, \tau \delta_{t} u_{N}^{k-(1 / 2)}\right)
\end{gathered}
$$

Applying Lemma 6 to the term in left side of (54), we obtain

$$
\begin{gathered}
\frac{\tau^{1-\alpha}}{c \Gamma(3-\alpha)} \sum_{k=1}^{m}\left\langle b_{0} \delta_{t} u_{N}^{k-(1 / 2)}-\sum_{l=1}^{k-1}\left(b_{l-1}-b_{l}\right) \delta_{t} u_{N}^{k-l-(1 / 2)}\right. \\
\left.-b_{k-1} v, \tau \delta_{t} u_{N}^{k-(1 / 2)}\right\rangle \\
\geq \frac{1}{2 c \Gamma(2-\alpha)} t_{m}^{1-\alpha} \tau \sum_{k=1}^{m}\left\|\delta_{t} u_{N}^{k-(1 / 2)}\right\|^{2} \\
-\frac{1}{2 c \Gamma(3-\alpha)} t_{m}^{2-\alpha}\|v\|^{2} .
\end{gathered}
$$

On the other side, based on the fact that $\delta_{t} u_{N}^{k-(1 / 2)}(a)=$ $\delta_{t} u_{N}^{k-(1 / 2)}(b)=0$, a straightforward calculation of the right terms in (54) gives

$$
\begin{aligned}
& \sum_{k=1}^{m}\left\langle\left(u_{N}^{k-(1 / 2)}\right)_{x x}, \tau \delta_{t} u_{N}^{k-(1 / 2)}\right\rangle \\
& =-\tau \sum_{k=1}^{m}\left\langle\left(u_{N}^{k-(1 / 2)}\right)_{x}, \delta_{t}\left(u_{N}^{k-(1 / 2)}\right)_{x}\right\rangle \\
& =-\frac{1}{2} \sum_{k=1}^{m}\left\langle\left(u_{N}^{k}\right)_{x}+\left(u_{N}^{k-1}\right)_{x},\left(u_{N}^{k}\right)_{x}-\left(u_{N}^{k-1}\right)_{x}\right\rangle \\
& =-\frac{1}{2}\left[\left\|\left(u_{N}^{m}\right)_{x}\right\|^{2}-\left\|\left(u_{N}^{0}\right)_{x}\right\|^{2}\right] . \\
& \sum_{k=1}^{m}\left\langle f^{k-(1 / 2)}, \tau \delta_{t} u_{N}^{k-(1 / 2)}\right\rangle \\
& \leq \frac{1}{2} \tau \sum_{k=1}^{m}\left[c \Gamma(2-\alpha) t_{m}^{\alpha-1}\left\|f^{k-(1 / 2)}\right\|^{2}\right. \\
& \left.+\frac{1}{c \Gamma(2-\alpha)} t_{m}^{1-\alpha}\left\|\delta_{t} u_{N}^{k-(1 / 2)}\right\|^{2}\right] .
\end{aligned}
$$

Substituting (55) and (56) into (54) yields inequality (53).

Suppose that $u^{k}, k=0,1,2, \ldots$, satisfy the conditions of Theorem 4 . Substituting the approximation solution $u_{N}^{k}$ given in (41) into (34) leads to

$$
\begin{gathered}
\frac{\tau^{1-\alpha}}{c \Gamma(3-\alpha)}\left[b_{0} \delta_{t} u_{N}^{k-(1 / 2)}-\sum_{l=1}^{k-1}\left(b_{l-1}-b_{l}\right) \delta_{t} u_{N}^{k-l-(1 / 2)}-b_{k-1} \psi\right] \\
=\left(u_{N}^{k-(1 / 2)}\right)_{x x}+\frac{1}{K} f^{k-(1 / 2)}+R_{N}^{k}, \quad k=1,2,3, \ldots
\end{gathered}
$$


Subtracting (57) from (34), we obtain

$$
\begin{aligned}
& \frac{\tau^{1-\alpha}}{c \Gamma(3-\alpha)} \\
& \quad \times\left(b_{0} \frac{1}{\tau}\left[\left(u^{k}-u_{N}^{k}\right)-\left(u^{k-1}-u_{N}^{k-1}\right)\right]\right. \\
& \left.\quad-\frac{1}{\tau} \sum_{l=1}^{k-1}\left(b_{l-1}-b_{l}\right)\left[\left(u^{k-l}-u_{N}^{k-l}\right)-\left(u^{k-l-1}-u_{N}^{k-l-1}\right)\right]\right) \\
& =\left(u^{k-(1 / 2)}\right)_{x x}-\left(u_{N}^{k-(1 / 2)}\right)_{x x}-R_{N}^{k}, \quad k=1,2,3, \ldots
\end{aligned}
$$

Based on Theorem 4, we have

$$
\begin{aligned}
R_{N}^{k}= & -\frac{1}{c \Gamma(3-\alpha) \tau^{\alpha}} \sqrt{n} e^{-\sqrt{\pi d \beta n}} \\
& \times\left[b_{0}\left(d_{k}-d_{k-1}\right)-\sum_{l=1}^{k-1}\left(b_{l-1}-b_{l}\right)\left(d_{k-l}-d_{k-l-1}\right)\right] \\
& +d^{k+1} n^{(3 / 2)} e^{-\sqrt{\pi d \beta n}}
\end{aligned}
$$

where $d_{0}, d_{1}, \ldots, d_{k+1}$ are constants.

Theorem 8. Let $u_{N}^{k}, k=0,1,2, \ldots$, be the approximation solution given in (41) and let $u^{k}$ satisfy the conditions of Theorem 4. Then one has

$$
\begin{aligned}
\left\|u_{N}^{k}\right\|^{2} \leq C & \left\|\left(u_{N}^{0}\right)_{x}\right\|^{2}+\frac{t_{k}^{2-\alpha}}{c \Gamma(3-\alpha)}\|\psi\|^{2} \\
& +c \Gamma(2-\alpha) t_{k}^{\alpha-1} \tau \\
& \left.\times \sum_{l=1}^{k}\left\|\frac{1}{K} f^{l-(1 / 2)}+R_{N}^{l-(1 / 2)}\right\|^{2}\right],
\end{aligned}
$$

where $C$ is constant.

Proof. Considering (57) and noting that $u_{N}^{0}=\varphi$ and $u_{N}^{k}(a)=$ $u_{N}^{k}(b)=0, k=0,1,2, \ldots$, we can easily obtain this result from Lemma 7 and the Poincaré inequality.

Theorem 9. Let the problem (19)-(21) have the exact solution $U^{k}$ and let $u_{N}^{k}$ be the approximation solution given in (41), where $k=0,1,2, \ldots$. If $u^{k}$ satisfy the conditions of Theorem 4, then for $k \tau \leq T$, one has

$$
\left\|e^{k}\right\| \leq \sqrt{\Gamma(2-\alpha) T^{\alpha}(b-a)}\left(c_{1} \tau^{3-\alpha}+c_{2} \tau^{-\alpha} n^{(3 / 2)} e^{-\sqrt{\pi d \beta n}}\right),
$$

where $e^{k}=U^{k}-u_{N}^{k}$.
Proof. Subtracting (57) from (32), we obtain the error equation

$$
\begin{aligned}
& \frac{\tau^{1-\alpha}}{c \Gamma(3-\alpha)}\left[b_{0} \delta_{t} e^{k-(1 / 2)}-\sum_{l=1}^{k-1}\left(b_{l-1}-b_{l}\right) \delta_{t} e^{k-l-(1 / 2)}\right] \\
& \quad=\left(e^{k-(1 / 2)}\right)_{x x}+R_{T}^{k}-R_{N}^{k},
\end{aligned}
$$

where $k=1,2,3, \ldots$ Noting that $e^{0}=0$ and $e^{k}(a)=e^{k}(b)=$ $0, k=1,2, \ldots$, and applying Lemma 7 , we have

$$
\left\|\left(e^{k}\right)_{x}\right\|^{2} \leq c \Gamma(2-\alpha) t_{k}^{\alpha-1} \tau \sum_{l=1}^{k}\left\|R_{T}^{l}-R_{N}^{l}\right\|^{2} .
$$

Based on (33) and (59), we obtain

$$
\begin{aligned}
\left\|\left(e^{k}\right)_{x}\right\| \leq & c_{3} \sqrt{\Gamma(2-\alpha) T^{\alpha}(b-a)} \\
& \times\left[\tau^{3-\alpha}+c_{4} \tau^{-\alpha} n^{(3 / 2)} e^{-\sqrt{\pi d \beta n}}\right] .
\end{aligned}
$$

Applying the Poincaré inequality yields the needed result.

Remark 10. In Theorem 9, the error estimate formula contains $c_{2} \tau^{-\alpha} n^{(3 / 2)} e^{-\sqrt{\pi d \beta n}}$ (the second term in the right-hand side), where the error contribution from the spatial approximation is affected by the inverse of the time step. It is worthwhile noting that similar results are also found for the classical diffusion/wave equation. However for large $n$, $n^{(3 / 2)} e^{-\sqrt{\pi d \beta n}}$ can be much smaller than $\tau$; therefore, this affection generally would not reduce the global accuracy.

\section{Numerical Examples}

To validate the effectiveness of the proposed method for the problem (19)-(21), we consider the example given in [18]. Consider the following:

$$
\begin{gathered}
\frac{\partial^{\alpha} u(x, t)}{\partial t^{\alpha}}=\frac{\partial^{2} u(x, t)}{\partial x^{2}}+\sin (\pi x), \quad 0<x<1,0<t \leq 1, \\
u(x, 0)=0, \quad \frac{\partial u(x, 0)}{\partial t}=0, \quad 0<x<1, \\
u(0, t)=0, \quad u(1, t)=0, \quad 0<t \leq 1 .
\end{gathered}
$$

The exact solution of the above problem is [33]

$$
u(x, t)=\frac{1}{\pi^{2}}\left[1-E_{\alpha}\left(-\pi^{2} t^{\alpha}\right)\right] \sin (\pi x),
$$

where $E_{\alpha}(z)=\sum_{k=0}^{\infty} z^{k} / \Gamma(\alpha k+1)$ which is one-parameter Mittag-Leffler function.

For solving the above problem (with $\alpha=1.3$ and 1.7, resp.) by using the method described in Section 3, we choose $\beta=1$ and $d=\pi / 2$, and this leads to $h=\pi / \sqrt{2 n}$. We will report the efficiency and accuracy of the given method based on the $L^{2}$-errors and $L^{\infty}$-errors. Figure 1 gives the 3D 


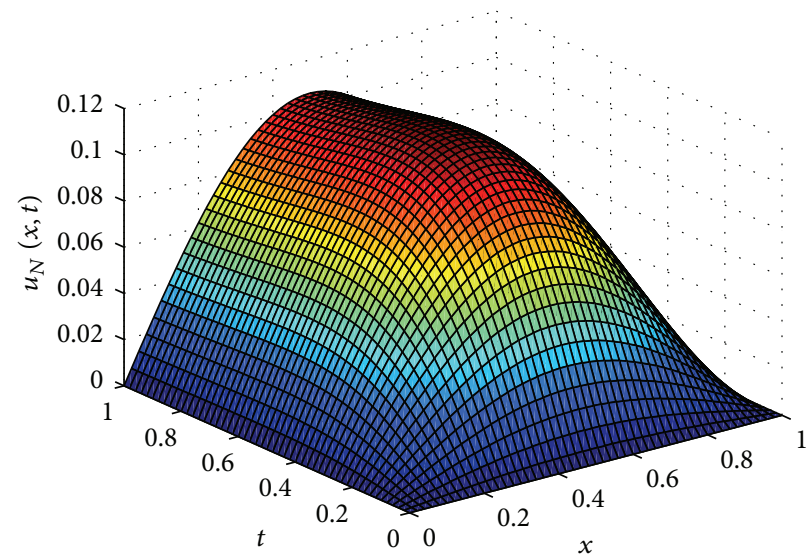

(a)

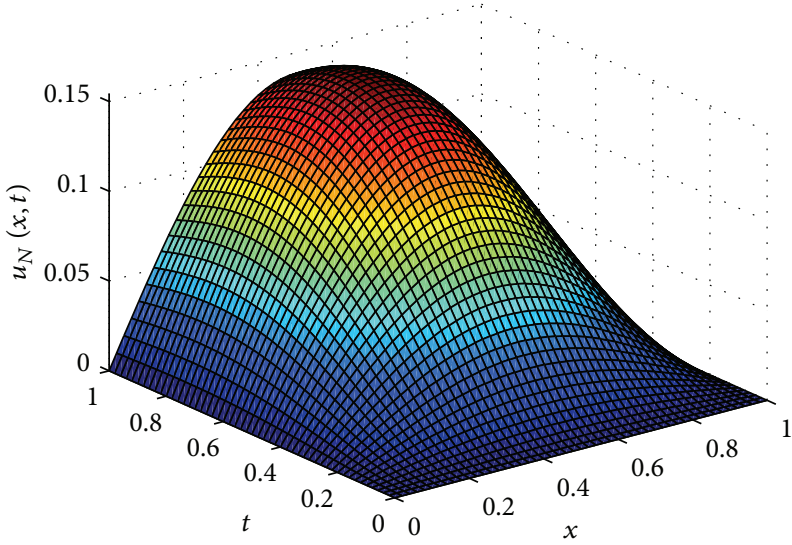

(b)

FIGURE 1: The numerical solutions with $\alpha=1.3$ (a) and $\alpha=1.7$ (b).

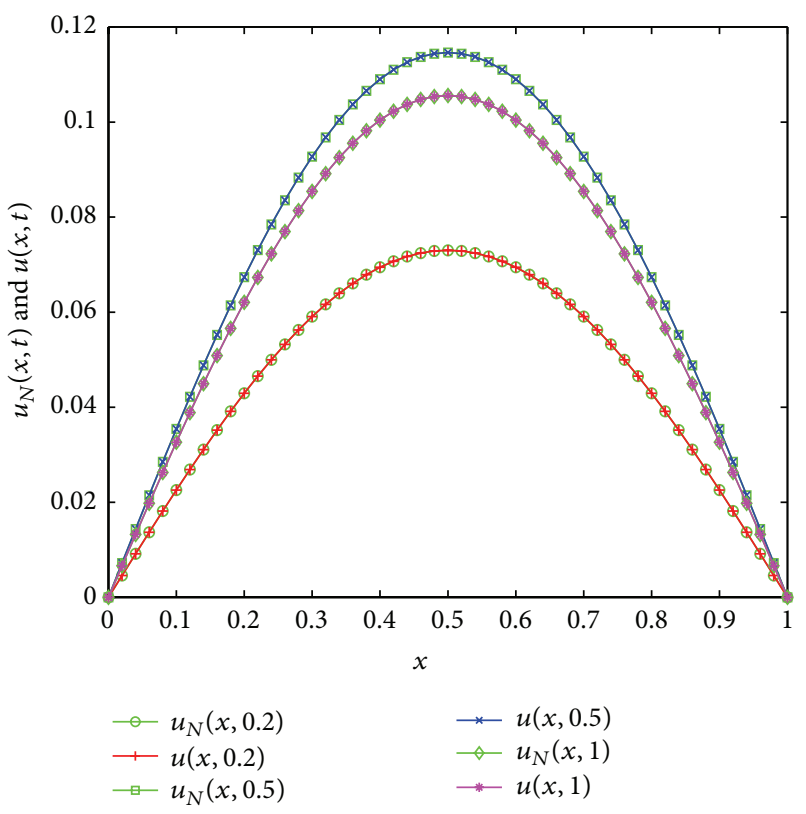

(a)

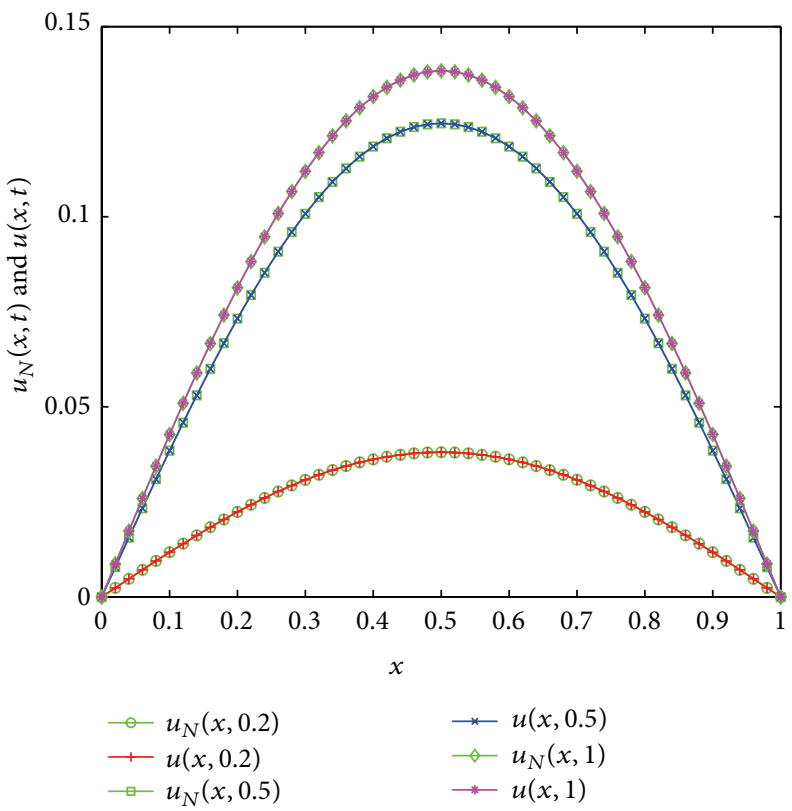

(b)

FIGURE 2: Comparison of the numerical and exact solutions for several fixed time instants with $\alpha=1.3$ (a) and $\alpha=1.7$ (b).

diagrams of the numerical solutions $u_{N}(x, t)$ on the whole computational domain $[0,1] \times[0,1]$ with $\tau=0.001, n=20$. In Figure 2, we plot the curves of the numerical solutions $u_{N}(x, t)$ and exact solutions $u(x, t)$ for several fixed time instants. A good agreement of the numerical solution with the exact one is achieved. Furthermore, Figure 3 shows the absolute error $\left|u_{N}(x, t)-u(x, t)\right|$ obtained by the present method with $\tau=0.001, n=20$.

To check the convergence behavior of the numerical solution with respect to the time step $\tau$ and the parameter $n$ about the number of collocation points, we represent the errors $\left\|U^{k}-u_{N}^{k}\right\|$ in two discrete norms: $L^{\infty}$ and $L^{2}$. All the numerical results reported in Figures 4 and 5, and Tables 1 and 2 are evaluated at $t=0.8$.
Firstly, the computational investigation is concerned with the temporal convergence rate. We choose the parameter $n=$ 60 and it is a value large enough such that the errors coming from the spatial approximation are negligible [35]. In Tables 1 $(\alpha=1.3)$ and $2(\alpha=1.7)$, we list the temporal errors when $\tau$ decreases from $1 / 10$ to $1 / 320$ and the convergence order which is very close to the expectation order $3-\alpha$. We also plot the errors in the $L^{\infty}$ and $L^{2}$ norms as a function of the time stepsize $\tau$ for several $\alpha$ in Figure 4, where a logarithmic scale has been used for both $\tau$-axis and error axis. From Figure 4, it is clear that, for $\alpha=1.3$ and 1.7, the slopes of the error curves in these log-log plots approach 1.7 and 1.3 , respectively. So the proposed method yields a temporal approximation order which is close to $3-\alpha$ as forecasted by the theoretical estimate. 


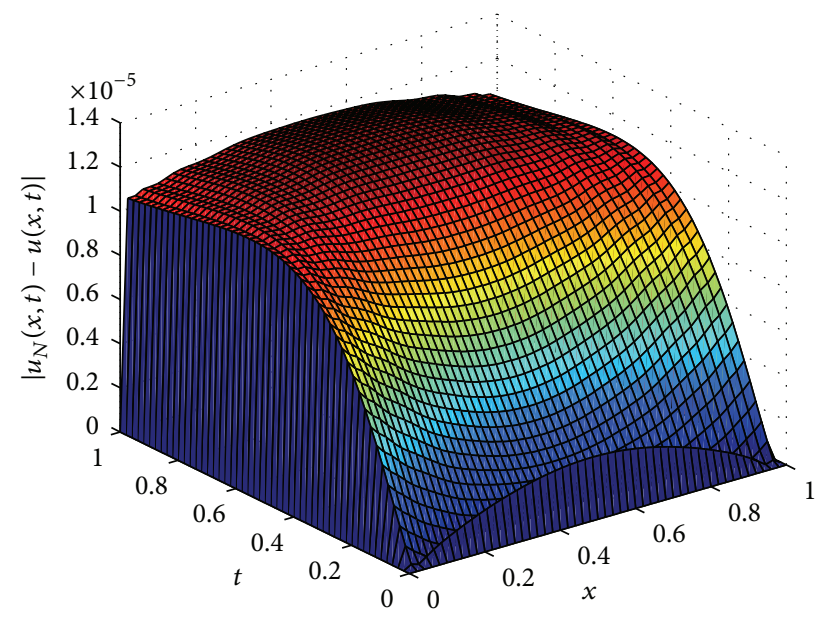

(a)

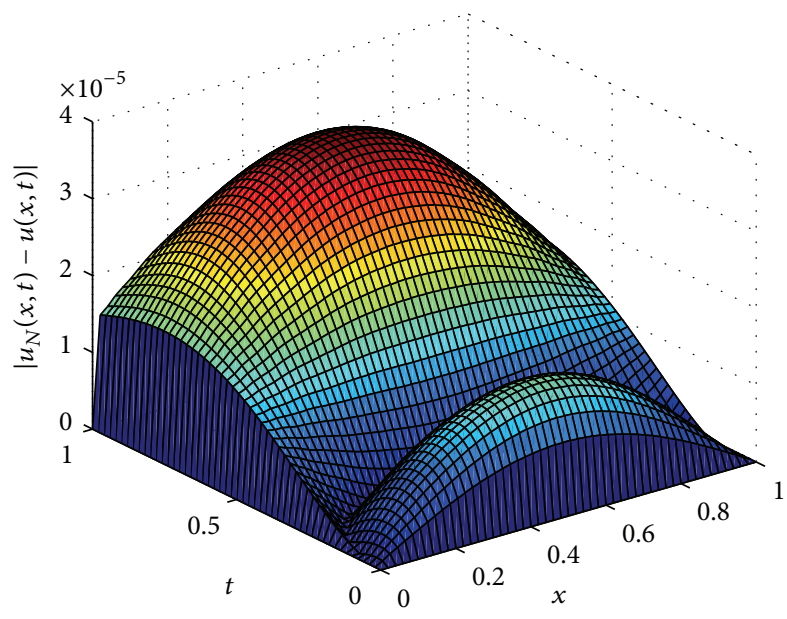

(b)

Figure 3: Plot of the absolute errors with $\alpha=1.3$ (a) and $\alpha=1.7$ (b).

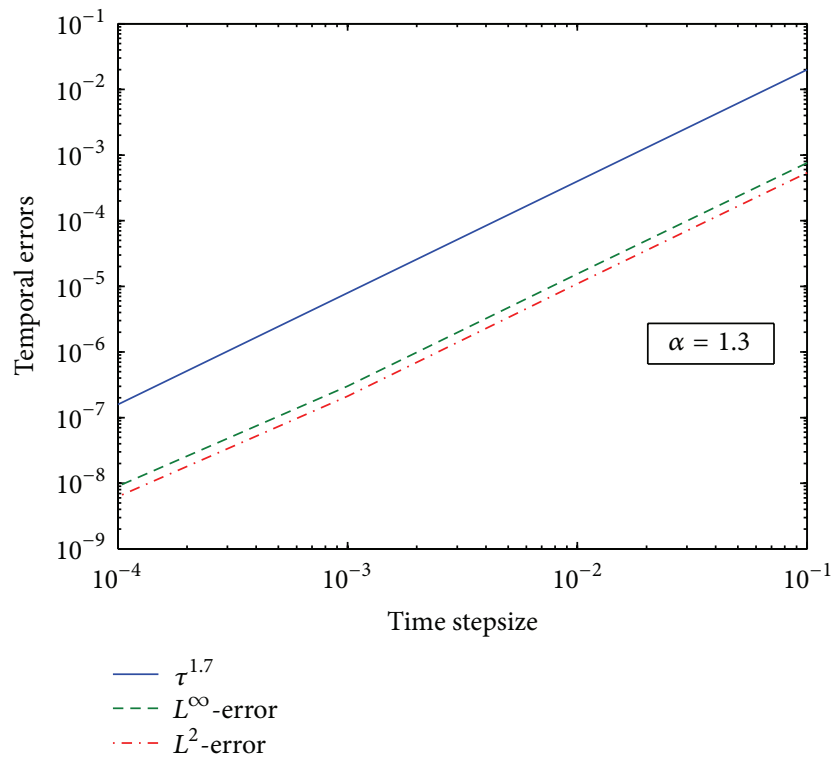

(a)

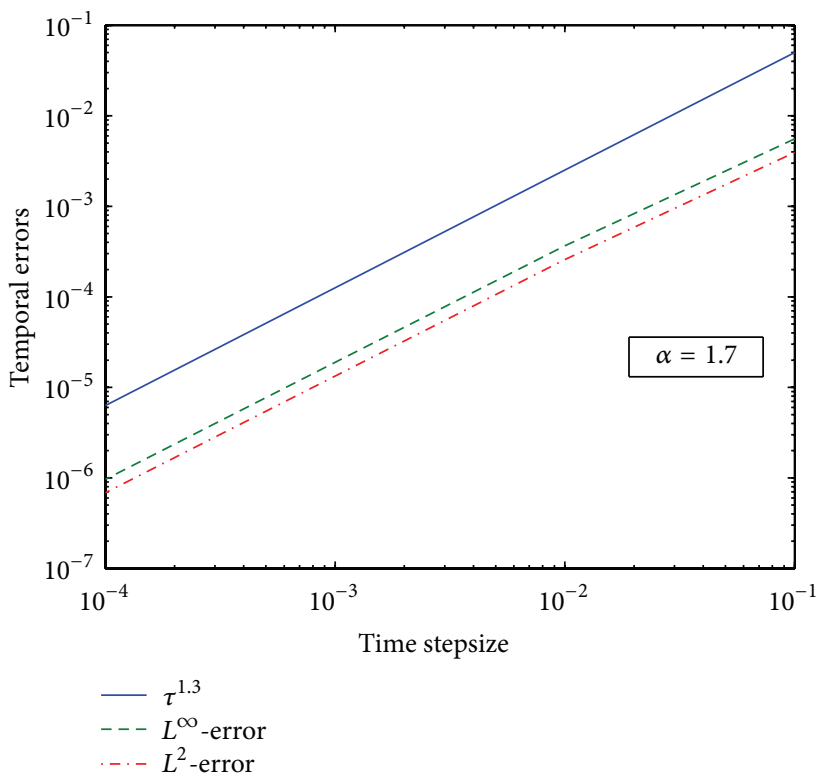

(b)

Figure 4: Temporal errors in the $L^{\infty}$ and $L^{2}$ norms versus $\tau$ for several $\alpha$.

Now we check the spatial accuracy with respect to the parameter $n$ about the number of collocation points. For the similar reason mentioned above, we fix the time step $\tau$ sufficiently small to avoid contamination of the temporal error. In Figure 5, we present the spatial errors as a function of $n$ with $\tau=0.0001$, where a logarithmic scale is now used for the spatial errors axis. It is clearly observed that the spatial errors appear in an exponential decay. In this semilog representations, we observe that the error variations are essentially linear versus $n$ [36].

\section{Conclusion}

In this paper, we have developed and analyzed an efficient numerical method for a class of the initial-boundary-value problems of fractional diffusion-wave equations. Based on a finite difference scheme in time and a global sinc-collocation method in space, the problem is reduced to the solution of a system of linear algebraic equations at each time step. The proposed method leads to $3-\alpha$ order accuracy in time and exponential convergence in space. The theoretical results are perfectly confirmed by the numerical experiments. 


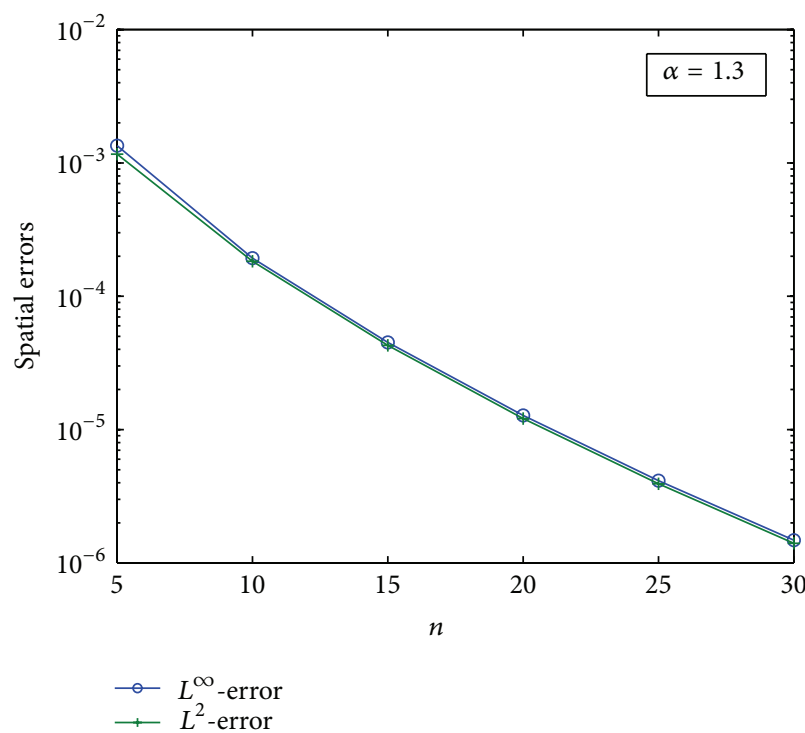

(a)

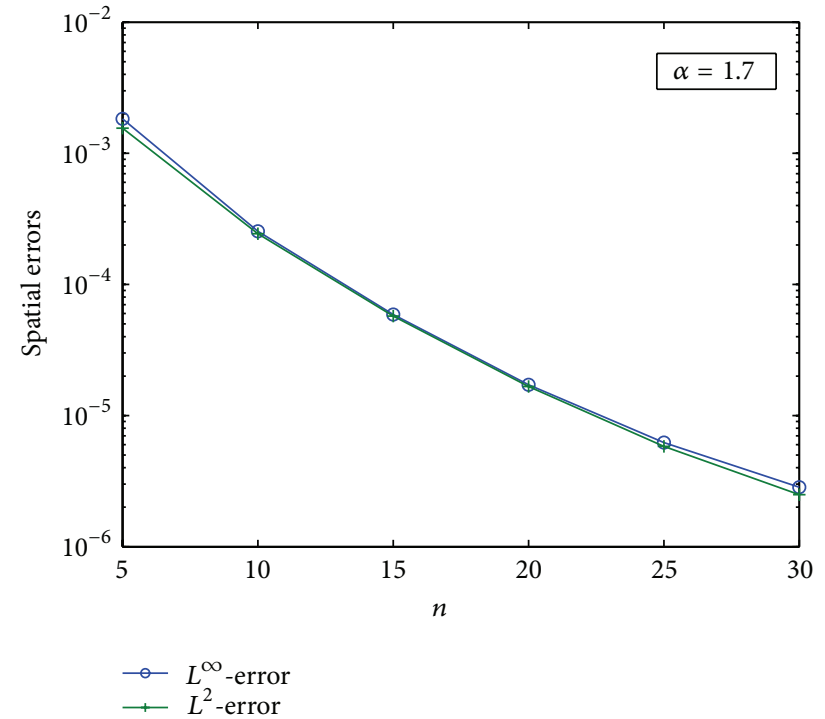

(b)

FIGURE 5: Spatial errors in the $L^{\infty}$ and $L^{2}$ norms versus $n$ for several $\alpha$.

TABLE 1: Temporal errors and convergence orders with $\alpha=1.3$.

\begin{tabular}{lcccc}
\hline$\tau$ & $L^{\infty}$-error & Order & $L^{2}$-error & Order \\
\hline $1 / 10$ & $7.589394 E-04$ & & $5.363813 E-04$ & \\
$1 / 20$ & $2.352470 E-04$ & 1.6898084 & $1.662599 E-04$ & 1.6898189 \\
$1 / 40$ & $7.279201 E-05$ & 1.6923243 & $5.144424 E-05$ & 1.6923586 \\
$1 / 80$ & $2.252242 E-05$ & 1.6924183 & $1.591604 E-05$ & 1.6925284 \\
$1 / 160$ & $6.961152 E-06$ & 1.6939638 & $4.918054 E-06$ & 1.6943215 \\
$1 / 320$ & $2.144986 E-06$ & 1.6983580 & $1.514220 E-06$ & 1.6995127 \\
$1 / 640$ & $6.553662 E-07$ & 1.7105947 & $4.614370 E-07$ & 1.7143693 \\
\hline
\end{tabular}

TABLE 2: Temporal errors and convergence orders with $\alpha=1.7$.

\begin{tabular}{lcccc}
\hline$\tau$ & $L^{\infty}$-error & Order & $L^{2}$-error & Order \\
\hline $1 / 10$ & $5.571817 E-03$ & & $3.937904 E-03$ & \\
$1 / 20$ & $2.625563 E-03$ & 1.0855211 & $1.855628 E-03$ & 1.0855201 \\
$1 / 40$ & $1.148662 E-03$ & 1.1926720 & $8.118237 E-04$ & 1.1926694 \\
$1 / 80$ & $4.844558 E-04$ & 1.2455178 & $3.423934 E-04$ & 1.2455116 \\
$1 / 160$ & $2.006883 E-04$ & 1.2714088 & $1.418397 E-04$ & 1.2713934 \\
$1 / 320$ & $8.239443 E-05$ & 1.2843377 & $5.823513 E-05$ & 1.2843000 \\
$1 / 640$ & $3.367394 E-05$ & 1.2909143 & $2.380175 E-05$ & 1.2908220 \\
\hline
\end{tabular}

\section{Conflict of Interests}

The authors declare that there is no conflict of interests regarding the publication of this paper.

\section{Acknowledgments}

The present research is supported by the National Natural Science Foundation of China (Grant nos. 11271311 and 11371016), the Chinese Program for Changjiang Scholars and Innovative Research Team in University (PCSIRT) (Grant no. IRT1179), and the Hunan Province Innovation Foundation for Postgraduate (Grant no. CX2013B252). The authors are grateful to the anonymous reviewers for their careful comments and valuable suggestions on this paper.

\section{References}

[1] F. Mainardi, "Fractional diffusive waves in viscoelastic solids," in Nonlinear Waves in Solids, J. L. Wegner and F. R. Norwood, Eds., pp. 93-97, ASME/AMR, Fairfield, NJ, USA, 1995.

[2] R. Balescu, "V-Langevin equations, continuous time random walks and fractional diffusion," Chaos, Solitons \& Fractals, vol. 34, no. 1, pp. 62-80, 2007.

[3] F. Mainardi, "Fractional relaxation-oscillation and fractional diffusion-wave phenomena," Chaos, Solitons \& Fractals, vol. 7, no. 9, pp. 1461-1477, 1996.

[4] A. A. Kilbas, H. M. Srivastava, and J. J. Trujillo, Theory and Applications of Fractional Differential Equations, Elsevier, Amsterdam, The Netherland, 2006.

[5] F. Mainardi, "The fundamental solutions for the fractional diffusion-wave equation," Applied Mathematics Letters, vol. 9, no. 6, pp. 23-28, 1996.

[6] S. Momani, Z. Odibat, and V. S. Erturk, "Generalized differential transform method for solving a space- and time-fractional diffusion-wave equation," Physics Letters A, vol. 370, no. 5-6, pp. 379-387, 2007.

[7] O. P. Agrawal, "Solution for a fractional diffusion-wave equation defined in a bounded domain," Nonlinear Dynamics, vol. 29, no. 1-4, pp. 145-155, 2002.

[8] Y. Luchko, "Fractional wave equation and damped waves," Journal of Mathematical Physics, vol. 54, no. 3, Article ID 031505, 16 pages, 2013.

[9] Y. Povstenko, "Axisymmetric solutions to fractional diffusionwave equation in a cylinder under Robin boundary condition," The European Physical Journal Special Topics, vol. 222, no. 8, pp. 1767-1777, 2013. 
[10] I. Podlubny, A. Chechkin, T. Skovranek, Y. Chen, and B. M. Vinagre Jara, "Matrix approach to discrete fractional calculus II: partial fractional differential equations," Journal of Computational Physics, vol. 228, no. 8, pp. 3137-3153, 2009.

[11] S. B. Yuste, "Weighted average finite difference methods for fractional diffusion equations," Journal of Computational Physics, vol. 216, no. 1, pp. 264-274, 2006.

[12] P. Zhuang, F. Liu, V. Anh, and I. Turner, "New solution and analytical techniques of the implicit numerical method for the anomalous subdiffusion equation," SIAM Journal on Numerical Analysis, vol. 46, no. 2, pp. 1079-1095, 2008.

[13] M. M. Khader, "On the numerical solutions for the fractional diffusion equation," Communications in Nonlinear Science and Numerical Simulation, vol. 16, no. 6, pp. 2535-2542, 2011.

[14] C. Piret and E. Hanert, "A radial basis functions method for fractional diffusion equations," Journal of Computational Physics, vol. 238, pp. 71-81, 2013.

[15] A. Mohebbi, M. Abbaszadeh, and M. Dehghan, "A high-order and unconditionally stable scheme for the modified anomalous fractional sub-diffusion equation with a nonlinear source term," Journal of Computational Physics, vol. 240, pp. 36-48, 2013.

[16] H. Jafari, A. Golbabai, S. Seifi, and K. Sayevand, "Homotopy analysis method for solving multi-term linear and nonlinear diffusion-wave equations of fractional order," Computers and Mathematics with Applications, vol. 59, no. 3, pp. 1337-1344, 2010.

[17] Z. M. Odibat and S. Momani, "Approximate solutions for boundary value problems of time-fractional wave equation," Applied Mathematics and Computation, vol. 181, no. 1, pp. 767774, 2006.

[18] Z. Sun and X. Wu, "A fully discrete difference scheme for a diffusion-wave system," Applied Numerical Mathematics, vol. 56, no. 2, pp. 193-209, 2006.

[19] J. Huang, Y. Tang, L. Vázquez, and J. Yang, "Two finite difference schemes for time fractional diffusion-wave equation," Numerical Algorithms, vol. 64, no. 4, pp. 707-720, 2013.

[20] J. Q. Murillo and S. B. Yuste, "An explicit difference method for solving fractional diffusion and diffusion-wave equations in the Caputo form," Journal of Computational and Nonlinear Dynamics, vol. 6, no. 2, Article ID 021014, 6 pages, 2011.

[21] F. Stenger, Numerical Methods Based on Sinc and Analytic Functions, Springer, New York, NY, USA, 1993.

[22] F. Stenger, Handbook of Sinc Numerical Methods, CRC Press, New York, NY, USA, 2011.

[23] J. Lund and K. L. Bowers, Sinc Methods f or Quadrature and Differential Equations, SIAM, Philadelphia, Pa, USA, 1992.

[24] K. Parand, M. Dehghan, and A. Pirkhedri, "The sinc-collocation method for solving the Thomas-Fermi equation," Journal of Computational and Applied Mathematics, vol. 237, no. 1, pp. 244-252, 2013.

[25] K. Al-Khaled, "Numerical study of Fisher's reaction-diffusion equation by the Sinc collocation method," Journal of Computational and Applied Mathematics, vol. 137, no. 2, pp. 245-255, 2001.

[26] A. Secer, "Numerical solution and simulation of second-order parabolic PDEs with Sinc-Galerkin method using Maple," Abstract and Applied Analysis, vol. 2013, Article ID 686483, 10 pages, 2013.

[27] M. Dehghan and F. Emami-Naeini, "The Sinc-collocation and Sinc-Galerkin methods for solving the two-dimensional
Schrödinger equation with nonhomogeneous boundary conditions," Applied Mathematical Modelling, vol. 37, no. 22, pp. 93799397, 2013.

[28] A. Saadatmandi, M. Dehghan, and M. Azizi, "The SincLegendre collocation method for a class of fractional convection-diffusion equations with variable coefficients," Communications in Nonlinear Science and Numerical Simulation, vol. 17, no. 11, pp. 4125-4136, 2012.

[29] G. Baumann and F. Stenger, "Fractional calculus and Sinc methods," Fractional Calculus and Applied Analysis, vol. 14, no. 4, pp. 568-622, 2011.

[30] I. Podlubny, Fractional Differential Equations, Academic Press, New York, NY, USA, 1999.

[31] D. Baleanu, K. Diethelm, E. S, and J. J. Trujillo, Fractional Calculus: Models and Numerical Methods, World Scientific, Singapore, 2012.

[32] M. Weilbeer, Efficient Numerical Methods for Fractional Differential Equations and their Analytical Background, Papierflieger, Clausthal-Zellerfeld, Germany, 2006.

[33] O. P. Agrawal, "Response of a diffusion-wave system subjected to deterministic and stochastic fields," Zeitschrift für Angewandte Mathematik und Mechanik, vol. 83, no. 4, pp. 265-274, 2003.

[34] K. Diethelm, The Analysis of Fractional Differential Equations, Springer, Berlin, Germany, 2010.

[35] Y. Lin and C. Xu, "Finite difference/spectral approximations for the time-fractional diffusion equation," Journal of Computational Physics, vol. 225, no. 2, pp. 1533-1552, 2007.

[36] X. Li and C. Xu, "A space-time spectral method for the time fractional diffusion equation," SIAM Journal on Numerical Analysis, vol. 47, no. 3, pp. 2108-2131, 2009. 


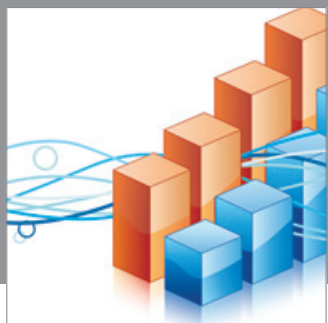

Advances in

Operations Research

mansans

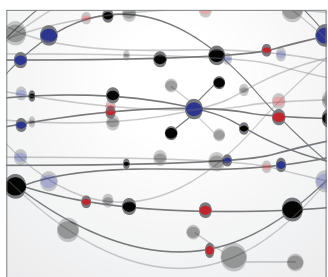

The Scientific World Journal
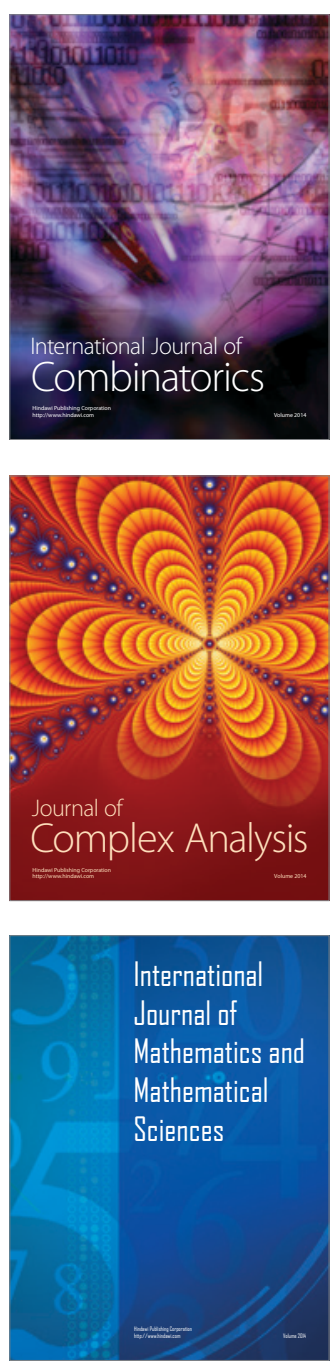
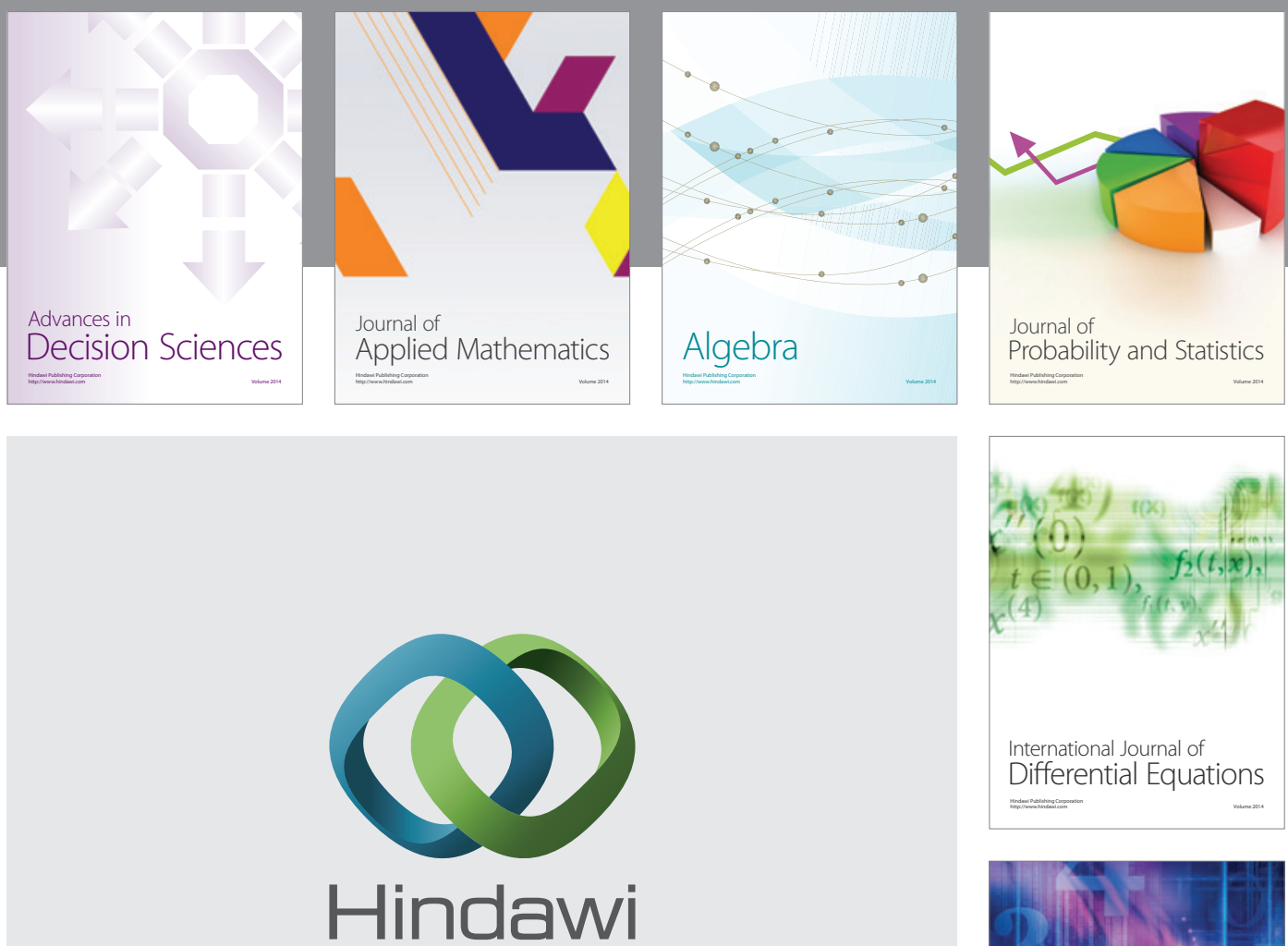

Submit your manuscripts at http://www.hindawi.com
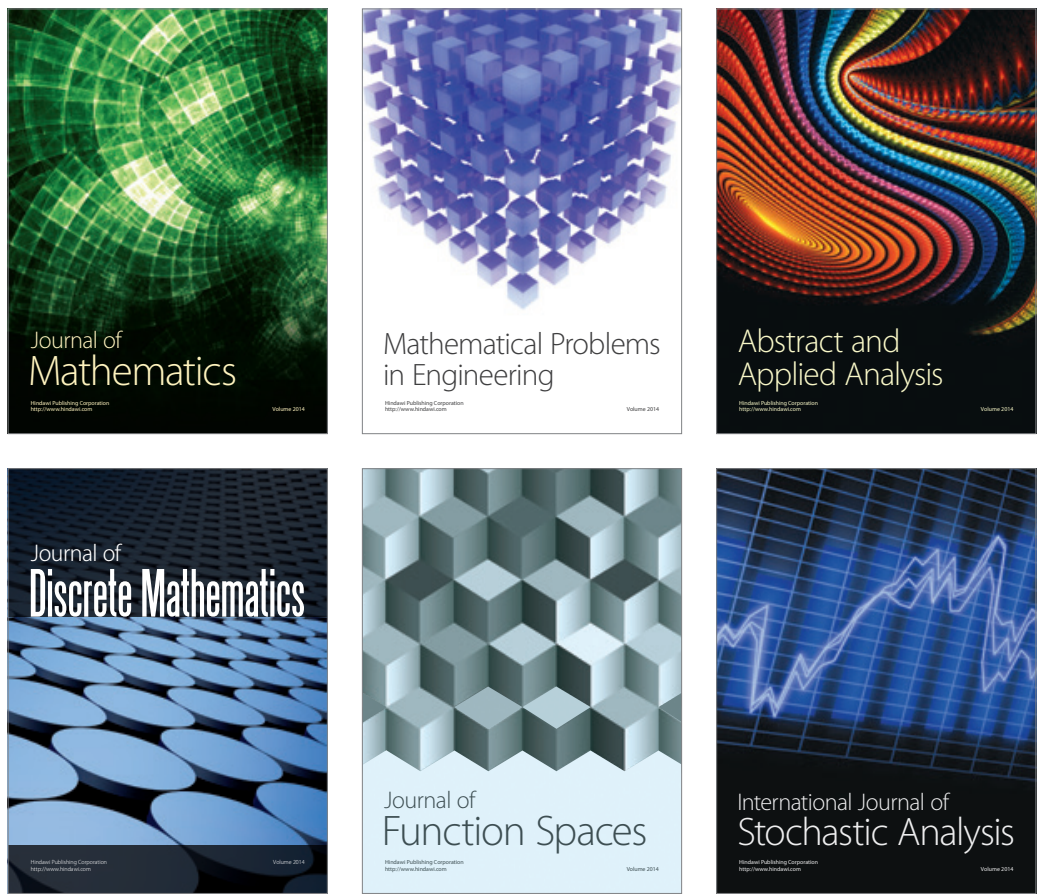

Journal of

Function Spaces

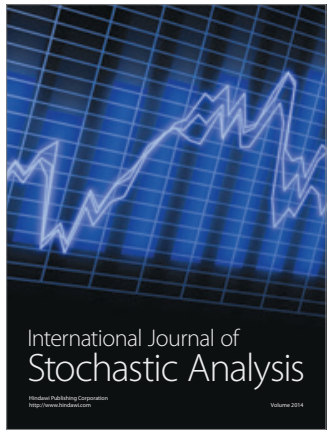

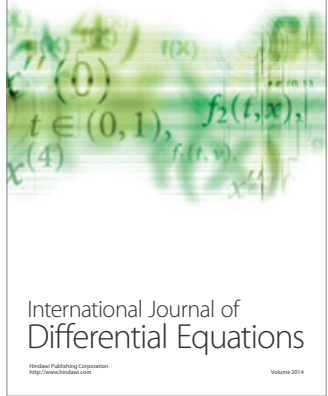
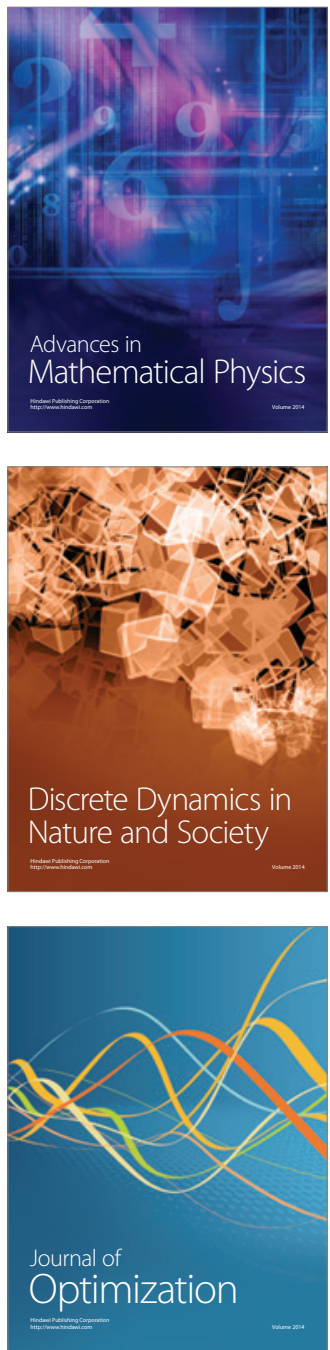